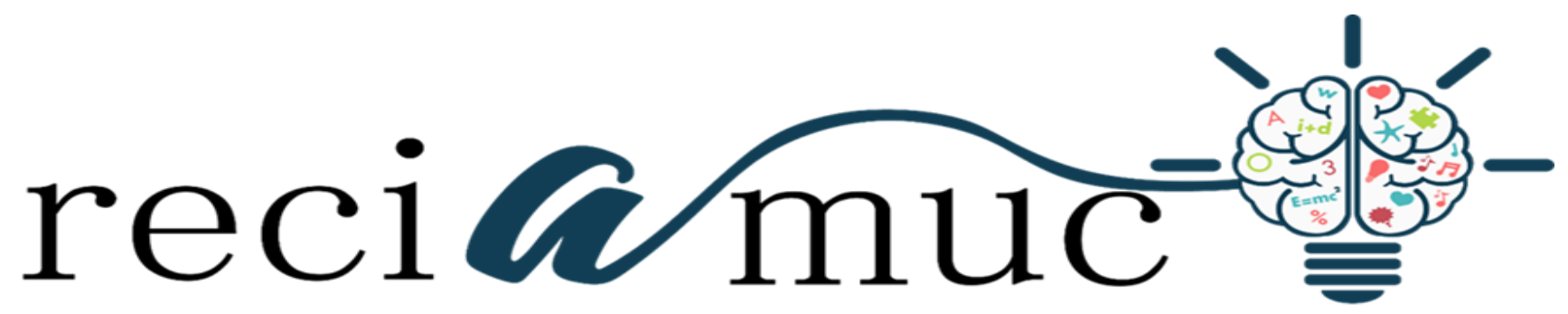

Revista cientifica de investigación actualización del mundo de las ciencias

Manuel Alejandro Cordones Sevillano a; Kenya Andreina Recalde Dicado ${ }^{\text {b; }}$ Shirley Natali Lema Sarmiento ${ }^{\mathrm{c}}$; Alex Renato Quimis Moran ${ }^{\mathrm{d}}$

El papel de los biomarcadores en el diagnóstico precoz de la endometriosis

The role of biomarkers in the early diagnosis of endometriosis

Revista Científica de Investigación actualización del mundo de las Ciencias. Vol. 3 núm., 2, abril, ISSN: 2588-0748, 2018, pp. 196-213

DOI: $10.26820 /$ reciamuc/3.(2).abril.2019.196-213

URL: http://reciamuc.com/index.php/RECIAMUC/article/view/333

Código UNESCO: 3205 Medicina Interna

Tipo de Investigación: Artículo de Revisión

(C) RECIAMUC; Editorial Saberes del Conocimiento, 2019

Recibido: 29/01/2019

Aceptado: 10/02/2019

Publicado: 01/04/2019

Correspondencia: cordonesmanuel@gmail.com
a. Médico; Saberes del Conocimiento; Guayaquil, Ecuador; cordonesmanuel@ gmail.com
b. Médico; Saberes del Conocimiento; Guayaquil, Ecuador; recaldekenya@gmail.com
c. Médico; Saberes del Conocimiento; Guayaquil, Ecuador; shirleylemast@gmail.com
d. Médico; Saberes del Conocimiento; Guayaquil, Ecuador; alexquimis@ hotmail.com 


\section{El papel de los biomarcadores en el diagnóstico precoz de la endometriosis}

Vol. 3, núm. 2., (2019)

Manuel Alejandro Cordones Sevillano; Kenya Andreina Recalde Dicado; Shirley Natali Lema Sarmiento; Alex Renato Quimis Moran

\section{RESUMEN}

La endometriosis es un problema ginecológico crónica que es frecuente en la población general. Debido a sus variadas presentaciones a menudo hay un gran retraso en el diagnóstico lo que conlleva a incurrir en una gran cantidad de daño psicológico y físico con la infertilidad como una de las desafortunadas secuelas. Si bien se han realizado mejoras en relación con el tratamiento de la endometriosis, el diagnóstico sigue siendo un reto para los médicos con las investigaciones tradicionales que producen malos resultados. Esta revisión considerará biomarcadores potenciales e investigaciones para el diagnóstico precoz de la endometriosis.

Palabras claves: Endometriosis, Diagnostico, Biomarcadores, Ginecología. 


\title{
El papel de los biomarcadores en el diagnóstico precoz de la endometriosis
}

Vol. 3, núm. 2., (2019)

Manuel Alejandro Cordones Sevillano; Kenya Andreina Recalde Dicado; Shirley Natali Lema

Sarmiento; Alex Renato Quimis Moran

\begin{abstract}
Endometriosis is a chronic gynecological problem that is common in the general population. Due to its varied presentations there is often a long delay in diagnosis, which leads to incurring a great deal of psychological and physical damage with infertility as one of the unfortunate sequels. While improvements have been made in relation to the treatment of endometriosis, diagnosis remains a challenge for doctors with traditional research that produces poor results. This review will consider potential biomarkers and investigations for the early diagnosis of endometriosis.
\end{abstract}

Key words: Endometriosis, Diagnosis, Biomarkers, Gynecology. 


\section{El papel de los biomarcadores en el diagnóstico precoz de la endometriosis}

Vol. 3, núm. 2., (2019)

Manuel Alejandro Cordones Sevillano; Kenya Andreina Recalde Dicado; Shirley Natali Lema

Sarmiento; Alex Renato Quimis Moran

\section{Introducción.}

La endometriosis es una condición ginecológica debilitante dependiente de estrógenos que se subdiagnostica en mujeres y se caracteriza por la implantación y adaptación exitosa de tejido endometrial en sitios extra pélvicos. Esto puede incluir el intestino, pulmones, uréteres y ovarios con un amplio espectro de severidad notado. Se cree que esta afección crónica afecta al 7-10\% de las mujeres de edad reproductiva y hasta la mitad de todas las mujeres infértiles (Grande, y otros 2017). Actualmente, debido a las insidiosas presentaciones de endometriosis en mujeres de edad fértil no es sorprendente que, en promedio, se necesita10.4 años desde el primer síntoma hasta el diagnóstico e incluso con las mejoras realizadas en las modalidades de imagen esto sigue siendo tan largo como 8 años. De hecho, esto puede causar varios problemas, incluida la necesidad para futura ooforectomía e histerectomía como resultado de una lesión progresiva (Oliveria, y otros 2017). Desafortunadamente en la mayoría, muchas mujeres son rechazadas y sus síntomas considerados de naturaleza funcional muchos años antes de que se haga este diagnóstico. Esto inevitablemente conduce a discapacidad grave y calidad de vida deteriorada. De hecho, debido a la naturaleza de esta condición muchas mujeres pueden pensar erróneamente que los síntomas que sufren son normales.

\section{Metodología.}

Para el desarrollo de este proceso investigativo, se plantea como metodología la encaminada hacia una orientación científica particular que se encuentra determinada por la necesidad de indagar en forma precisa y coherente una situación, en tal sentido (Davila, 2015) 


\section{El papel de los biomarcadores en el diagnóstico precoz de la endometriosis}

Vol. 3, núm. 2., (2019)

Manuel Alejandro Cordones Sevillano; Kenya Andreina Recalde Dicado; Shirley Natali Lema Sarmiento; Alex Renato Quimis Moran

define la metodología "como aquellos pasos previos que son seleccionados por el investigador para lograr resultados favorables que le ayuden a plantear nuevas ideas”. (p.66)

Lo citado por el autor, lleva a entender que el desarrollo de la acción investigativa busca simplemente coordinar acciones enmarcadas en una revisión bibliográfica con el fin de complementar ideas previas relacionadas al uso de biomarcadores en el diagnóstico de la endometriosis a través de una revisión de literatura, para así finalmente elaborar un cuerpo de consideraciones generales que ayuden a ampliar el interés propuesto.

\section{Tipo de Investigación.}

Dentro de toda práctica investigativa, se precisan acciones de carácter metodológico mediante las cuales, se logra conocer y proyectar los eventos posibles que la determinan, así como las características que hacen del acto científico un proceso interactivo ajustado a una realidad posible de ser interpretada. En este sentido, se puede decir, que la presente investigación corresponde al tipo documental, definido por Castro (2016), "se ocupa del estudio de problemas planteados a nivel teórico, la información requerida para abordarlos se encuentra básicamente en materiales impresos, audiovisuales y /o electrónicos”. (p.41).

En consideración a esta definición, la orientación metodológica permitió la oportunidad de cumplir con una serie de actividades inherentes a la revisión y lectura de diversos documentos donde se encontraron ideas explicitas relacionadas con los tópicos encargados de identificar a cada característica insertada en el estudio. Por lo tanto, se realizaron continuas interpretaciones con el claro propósito de revisar aquellas apreciaciones o investigaciones propuestas por diferentes 


\section{El papel de los biomarcadores en el diagnóstico precoz de la endometriosis}

Vol. 3, núm. 2., (2019)

Manuel Alejandro Cordones Sevillano; Kenya Andreina Recalde Dicado; Shirley Natali Lema Sarmiento; Alex Renato Quimis Moran

investigadores relacionadas con el tema de interés, para luego dar la respectiva argumentación a los planteamientos, en función a las necesidades encontradas en la indagación.

\section{Fuentes Documentales.}

El análisis correspondiente a las características que predomina en el tema seleccionado, llevan a incluir diferentes fuentes documentales encargadas de darle el respectivo apoyo y en ese sentido cumplir con la valoración de los hechos a fin de generar nuevos criterios que sirven de referencia a otros procesos investigativos. Para (CASTRO, 2016) las fuentes documentales incorporadas en la investigación documental o bibliográfica, "representa la suma de materiales sistemáticos que son revisados en forma rigurosa y profunda para llegar a un análisis del fenómeno".(p.41). Por lo tanto, se procedió a cumplir con la realización de una lectura previa determinada para encontrar aquellos aspectos estrechamente vinculados con el tema, con el fin de explicar mediante un desarrollo las respectivas apreciaciones generales de importancia.

\section{Técnicas para la Recolección de la Información.}

La conducción de la investigación para ser realizada en función a las particularidades que determinan a los estudios documentales, tiene como fin el desarrollo de un conjunto de acciones encargadas de llevar a la selección de técnicas estrechamente vinculadas con las características del estudio. En tal sentido, (Bolívar, 2015), refiere, que es "una técnica particular para aportar ayuda a los procedimientos de selección de las ideas primarias y secundarias”. (p. 71).

Por ello, se procedió a la utilización del subrayado, resúmenes, fichaje, como parte básica para la revisión y selección de los documentos que presentan el contenido teórico. Es decir, que 


\section{El papel de los biomarcadores en el diagnóstico precoz de la endometriosis}

Vol. 3, núm. 2., (2019)

Manuel Alejandro Cordones Sevillano; Kenya Andreina Recalde Dicado; Shirley Natali Lema Sarmiento; Alex Renato Quimis Moran

mediante la aplicación de estas técnicas se pudo llegar a recoger informaciones en cuanto a la revisión bibliográfica de los diversos elementos encargados de orientar el proceso de investigación. Tal como lo expresa, (Bolívar, 2015) "las técnicas documentales proporcionan las herramientas esenciales y determinantes para responder a los objetivos formulados y llegar a resultados efectivos" (p. 58). Es decir, para responder con eficiencia a las necesidades investigativas, se introdujeron como técnica de recolección el método inductivo, que hizo posible llevar a cabo una valoración de los hechos de forma particular para llegar a la explicación desde una visión general.

Asimismo, se emplearon las técnicas de análisis de información para la realización de la investigación que fue ejecutada bajo la dinámica de aplicar diversos elementos encargados de determinar el camino a recorrer por el estudio, según, (Bolívar, 2015) las técnicas de procesamiento de datos en los estudios documentales "son las encargadas de ofrecer al investigador la visión o pasos que debe cumplir durante su ejercicio, cada una de ellas debe estar en correspondencia con el nivel a emplear" (p. 123). Esto indica, que para llevar a cabo el procesamiento de los datos obtenidos una vez aplicado las técnicas seleccionadas, tales como: fichas de resumen, textual, registros descriptivos entre otros, los mismos se deben ajustar al nivel que ha sido seleccionado.

\section{Resultados.}

En la actualidad, hay tres tipos de endometriosis reconocidas; infiltración superficial, ovárica y profunda. Lesiones superficiales son probablemente de naturaleza pélvica y pueden identificarse como azul-negro en observación directa. Depósitos de endometriosis ovárica también conocidas como "quistes de chocolate" ya que los endometriomas son psuedoquistes que son 


\section{El papel de los biomarcadores en el diagnóstico precoz de la endometriosis}

Vol. 3, núm. 2., (2019)

Manuel Alejandro Cordones Sevillano; Kenya Andreina Recalde Dicado; Shirley Natali Lema Sarmiento; Alex Renato Quimis Moran

mucho más probable que forme adherencias a las estructuras circundantes. Sin embargo, la endometriosis infiltrante profunda se define como la extensión de glándulas endometriales $>5 \mathrm{~mm}$ debajo de la superficie peritoneal y la mayoría con frecuencia involucra los ligamentos uterosacros y está asociado conadenomiosis subyacente. Estas lesiones están clásicamente relacionadas con los pacientes con dolor cíclico y son masas sólidas de tejido del endometrio tejido con proliferación de músculo liso y fibroso asociado (Hsu, y otros 2011).

Los síntomas iniciales de la endometriosis incluyen dismenorrea, dispareunia, dolor abdominal bajo, disuria y habito de intestino alterado. De estos, la dismenorrea es el síntoma más común y agravando esto hasta el $20 \%$ de las mujeres afectadas tendrán otras condiciones de dolor crónico que incluyen fibromialgia, migraña y síndrome del intestino irritable. Se debe a esta presentación variada que los médicos a menudo tienen que confiar en una buena historia clínica, signos y síntomas para hacer el diagnóstico. Una revisión sistemática que aborda la utilidad de los signos / síntomas en el diagnóstico de la endometriosis mostró que la dismenorrea y la dispareunia están universalmente presente en la endometriosis particularmente en la etapa temprana de la enfermedad (Riazi, y otros 2015). Los puntos clave dentro de la historia incluyen la presencia de síntomas y su momento dentro del ciclo menstrual de las mujeres, la duración del ciclo menstrual de las mujeres y la regularidad de sus períodos. Sin embargo, mientras que el examen clínico esencial del paciente a menudo proporciona poca información con hallazgos como desviación cervical, sensibilidad cervical; útero retrovertido fijo omasa anexial a menudo no encontrada. Pero cuando se encuentra es más probable que sea presente cuando el examen se realiza durante la menstruación. 


\section{El papel de los biomarcadores en el diagnóstico precoz de la endometriosis}

Vol. 3, núm. 2., (2019)

Manuel Alejandro Cordones Sevillano; Kenya Andreina Recalde Dicado; Shirley Natali Lema Sarmiento; Alex Renato Quimis Moran

La comprensión actual es que esta condición tiene tanto una genética y componentes ambientales con pacientes que tienen un pariente de primer grado afectado tienen hasta 6 veces el riesgo relativo de desarrollar la condición frente a los controles (Grande, y otros 2017). De hecho, la opinión actual es que el $51 \%$ de la variación en el riesgo de endometriosis es heredable. Sin embargo, estudios para investigar la base genética de la endometriosis no han reproducido los genes candidatos. Recientemente, sin embargo, un estudio de asociación del genoma completo (GWAS) utilizando pacientes con endometriosis de tres continentes han demostrado que hay 10 loci que en conjunto sólo representan $\sim 5 \%$ de la heredabilidad de endometriosis subyacente (A, y otros 2015). Se requiere, como tal, mucho más trabajo con respecto a la metilación del ADN y la modificación de las histonas.

Históricamente, la patofisiología de la endometriosis se ha explicado por la teoría Sampsons de la menstruación retrógrada secundaria a reflujo trompa de Falopio (Guerriero, y otros 2015). Si bien esto ha formado gran parte de la base de la investigación endometrial y entender el hecho de que una escasez de técnicas de diagnóstico para esta condición existe sigue siendo evidente. De hecho, incluso después de varias décadas de investigación sigue siendo el estándar de oro en laparoscopia que incurre en una gran estética, daños psicológicos y físicos, pero puede identificar todos los tipos de endometriosis (Guerriero, y otros 2015). Sin embargo, incluso esta regla de oro tiene defectos potenciales con diagnóstico visual de endometriosis en este proceso demostrando ser subóptima. Se cree que hasta la mitad de todos los resultados positivos en la laparoscopia será incorrecta con diferenciales incluyendo cambios inflamatorios benignos (Hsu, y otros 2011). 


\section{El papel de los biomarcadores en el diagnóstico precoz de la endometriosis}

Vol. 3, núm. 2., (2019)

Manuel Alejandro Cordones Sevillano; Kenya Andreina Recalde Dicado; Shirley Natali Lema Sarmiento; Alex Renato Quimis Moran

Como la laparoscopia representa un método extremo de diagnóstico de la enfermedad debido a su alta frecuencia dentro de la población, los médicos a menudo inicialmente confían en técnicas de imagen comunes y fácilmente accesibles, tales como la ecografía transvaginal (ETV). La ETV es una modalidad común que se utiliza dentro de la ginecología para el diagnóstico de una miríada de problemas. En una reciente revisión sistemática mirando a la exactitud de esta modalidad que se encontró que tenía una sensibilidad del 53\%, 49\% y 58\% en uterinesacral, tabique recto vaginal y la endometriosis vaginal, respectivamente (Tadros y Keriakos 2016). De hecho, el problema con ETV es que a menudo el detalle que proporciona es subóptima en particular para la infiltración profunda lesiones endometriales y no es sensible para grandes áreas de enfermedad. A medida que cada vez se utiliza como MRI por esta razón, así como para el diagnóstico de endometriomas, estadio de la enfermedad y confirmar la presencia de adherencias. Todo esto se puede lograr a intensidades de campo magnético de 3.0T . El consenso general en la actualidad es que la RM es más sensible cuando se realiza durante la primera mitad del ciclo menstrual con la vejiga llena para eliminar la anteversión del útero. Que utilizan la difusión de imágenes ponderada junto al coeficiente de difusión aparente (ADC) los valores de los radiólogos han sido capaces de identificar con precisión y grandes endometriomas sólidos. Esto es porque demuestran patrones de difusión restringida frente a otras patologías pélvicas incluyendo quistes hemorrágicos y quistes dermoides. En la actualidad la mayoría de los protocolos de formación de imágenes sugieren que las secuencias ponderadas en T1 se pueden utilizar para detectar lesiones endometriales de $<1 \mathrm{~cm}$ con secuencias de supresión grasa que permite la diferenciación de hemorragias y lesiones quísticas. En comparación las lesiones fibrosas puras exhibirán intensidad de formación de imágenes bajo T1 / T2, mientras que la mayoría de las lesiones endometriales 


\section{El papel de los biomarcadores en el diagnóstico precoz de la endometriosis}

Vol. 3, núm. 2., (2019)

Manuel Alejandro Cordones Sevillano; Kenya Andreina Recalde Dicado; Shirley Natali Lema Sarmiento; Alex Renato Quimis Moran

muestran una baja intensidad en las imágenes T2. Como tal, la especificidad informada de MRI puede ser tan alta como 97\% (Das y Bast 2008)

Sin embargo, como resonancia magnética sigue siendo un medio difícil y costoso para el diagnóstico de una afección tan común que se mantiene la esperanza de que una prueba no invasiva se puede hacer utilizando la orina, sangre, líquido peritoneal, etc., que con alta sensibilidad identifica enfermedad endometrial temprano en la presencia de un ETV normal. En efecto, mediante el desarrollo de una prueba de este tipo, se podría buscar pronosticar las mujeres en función de sus niveles de dichos biomarcadores. Un biomarcador que ha tenido una gran cantidad de atención es CA-125. Esta glicoproteína musinosa se expresa por los derivados de epitelio celómico y de Müller y puede ser regulada por incremento tanto en el suero y el líquido peritoneal en muchas condiciones ginecológicas, tanto benignas como malignas en la naturaleza donde se produce la inflamación peritoneal (Gupta, y otros 2006). Los primeros estudios de CA-125 en esta configuración ha demostrado que la sensibilidad de este marcador disminuye con etapas más avanzadas de la enfermedad y que los pacientes con endometriosis han aumentado significativamente las concentraciones de CA-125 en el líquido peritoneal frente a los controles con independencia de la fase de la menstruación. Un meta-análisis de los resultados de CA-125 en esta configuración reveló que la sensibilidad de este marcador oscila entre 4-100\% con una especificidad del 38-100\%. De hecho, al igual que en el cáncer de ovario en donde se utiliza este biomarcador, se piensa que se puede utilizar mejor cuando se usa como parte de un panel de biomarcadores, con la sensibilidad de este marcador a la mejora de 74-94\% con una especificidad del 55-75\% cuando se combina con VEGF, anexina V y sICAM1 (Guerriero, y otros 2015). Sin embargo, al tiempo que promete estos resultados necesitan ser ratificado de acuerdo con la edad y 


\section{El papel de los biomarcadores en el diagnóstico precoz de la endometriosis}

Vol. 3, núm. 2., (2019)

Manuel Alejandro Cordones Sevillano; Kenya Andreina Recalde Dicado; Shirley Natali Lema Sarmiento; Alex Renato Quimis Moran

origen étnico de las cohortes de mujeres, ambos de los cuales se sabe que influyen CA-125 valores. Además, el uso de este biomarcador permanece subóptimo en el diagnóstico de endometriosis debido a su baja especificidad. Otros biomarcadores séricos que mantienen la promesa en el futuro incluyen factores clave que median la angiogénesis y que incluyen el factor de crecimiento de fibroblastos 2 (FGF2) y endoglina que también puede tener usos en el monitoreo progresión de la endometriosis y de su respuesta al tratamiento (Mol, y otros 1998).

A diferencia de otros tejidos del cuerpo el endometrio humano es aquel que de manera coordinada cambia su composición y arquitectura sobre una base mensual. Este proceso es uno que ve una gran proliferación y la hormona de secreción celular y retirada de la hormona eventual y la apoptosis. Este proceso se sostiene la angiogénesis, que es un proceso clave en la patogénesis de la endometriosis. En el estudio de este proceso se ha puesto de manifiesto que los microRNAs (miRNAs) son fundamentales en este. Los microARN se codifican con los genes implicados en la regulación de la expresión génica y por lo tanto controlan fundamentalmente los sistemas biológicos a través de silenciamiento de genes de varios miRNAs simultáneamente. A través de mejoras en la tecnología de secuenciación de próxima generación y de microarrays que ahora son capaces de estudiar los efectos de miRNAs en una escala genómica. Además, la presencia de circulación miARN en la sangre ha conducido a la investigación de su aplicación como biomarcadores en varias condiciones incluyendo sepsis y el infarto de miocardio. Las primeras investigaciones en este campo han revelado expresiones miARN diferenciales entre los pacientes y los controles de endometrio con regulación a la baja de miR-34C-5p, miR-9 y miR-34b, let-7d etc. Let-7d se descubrió el primer miARN para promover la diferenciación celular con reducción de expresión de let-7, miembros de la familia bien conocida por estar asociada con la malignidad 


\section{El papel de los biomarcadores en el diagnóstico precoz de la endometriosis}

Vol. 3, núm. 2., (2019)

Manuel Alejandro Cordones Sevillano; Kenya Andreina Recalde Dicado; Shirley Natali Lema Sarmiento; Alex Renato Quimis Moran

que ha demostrado tener la sensibilidad endometriosis y especificidad de $83,3 \%$ y $100 \%$, respectivamente (Cosar, y otros 2016). Es comprensible que la regulación a la baja de un miARN conduce a un aumento de su correspondiente Las primeras investigaciones en este campo ha revelado expresiones miARN diferenciales entre los pacientes y los controles de endometrio con regulación a la baja de miR-34C-5p, miR-9 y miR-34b, let-7d etc. Let-7d se descubrió el primer miARN para promover la diferenciación celular con reducción de expresión de let-7 miembros de la familia bien conocida por estar asociada con la malignidad que ha demostrado tener la sensibilidad endometriosis y especificidad de $83,3 \%$ y $100 \%$, respectivamente (Cosar, y otros 2016).

Comprensiblemente la regulación a la baja de un miARN conduce a un aumento de su producto genético de ARNm correspondiente que regula, muchos de los cuales han sido implicado en inflamación, mitosis, señalización celular, angiogénesis y remodelación extracelular.

Actualmente, miR-125-5P se considera el más efectivo marcador de diagnóstico en la endometriosis que se regula en este ajuste cuando se mide por PCR cuantitativa en tiempo real y se analiza utilizando las características de operación del receptor. Además, tenía la mayor sensibilidad y especificidad de cualquier miARN en pacientes de cirugía diagnosticados. De hecho, cuando se combina con miR-451a ymIR-3613-5p se alcanzó una puntuación máxima de AUC 1.00 (Borghese, y otros 2016).

Una pregunta clave sigue siendo qué causa esta desregulación en el primer lugar. Esto se debe a que se sabe que la expresión de miRNA se ve afectada por edad, etnia y actividad subyacente de la enfermedad. El último de estos es un punto importante ya que cada uno de los 


\section{El papel de los biomarcadores en el diagnóstico precoz de la endometriosis}

Vol. 3, núm. 2., (2019)

Manuel Alejandro Cordones Sevillano; Kenya Andreina Recalde Dicado; Shirley Natali Lema Sarmiento; Alex Renato Quimis Moran

tres tipos de endometriosis exhibe un perfil de miARN diferente que se ve afectado por el punto del ciclo menstrual en el que se miden con un circadianoritmo de expresión que se cree que existe. Debido a esto y a los pequeños tamaños de estudio utilizados hasta la fecha, así como la escasez de modelos establecidos en el que la investigación de miARN se puede realizar in vivo, hay mucho que hacer antes de realizar cualquier traducción a la clínica.

Debido a la presencia de tejido endometrial ectópico es de no sorprender que pueda haber un componente inmunológicoa esta enfermedad. Se ha considerado que la endometriosis puede desarrollarse como resultado de la eliminación inadecuada del tejido ectópico debido a las alteraciones subyacentes de las células inmunes, incluida la disminución de CD36y expresión de LFA-1 y citotoxicidad disminuida de linfocitos $\mathrm{T}$. De hecho, se piensa que las alteraciones hormonales en la endometriosis para perpetuar este estado proinflamatorio. Por otra parte, ha sido reconocido que los pacientes afectados muestran inmunidad alterada y, a menudo, tienen una mayor prevalencia de autoinmunidad a la enfermedad que incluye lupus eritematoso sistémico (LES). Ahora, Se han demostrado varios autoanticuerpos en el suero de pacientes endometriales que incluyen anti- $\alpha$-enolasa, anti-PDKI1L, antisyntaxina5 autoanticuerpos (Randall, y otros 2007). De hecho, el anticuerpo anti endometrialha demostrado tener un valor predictivo positivo del $88 \%$ cuando es utilizado antes de la laparoscopia en el diagnóstico de endometriosis. Sin embargo, aunque medir los títulos inmunológicos es útil, la preocupación es el hecho de que estos autoanticuerpos se pueden encontrar en mujeres y hombres no afectados. Además, no se han realizado cortes asignado para discriminar entre enfermedad activa e inactiva lo que significa que ofrecen poca información o pronóstico de los afectados individuos. 


\section{El papel de los biomarcadores en el diagnóstico precoz de la endometriosis}

Vol. 3, núm. 2., (2019)

Manuel Alejandro Cordones Sevillano; Kenya Andreina Recalde Dicado; Shirley Natali Lema Sarmiento; Alex Renato Quimis Moran

Si bien los marcadores séricos siguen siendo importantes, los cambios en la ultraestructura del endometrio podrían ofrecer importantes ideas sobre la enfermedad endometrial temprana. En los últimos años ha habido un interés considerable con respecto a la biopsia nerviosa del endometrio. Hace más de una década se observó por primera vez que pacientes con endometriosis tienen múltiples pequeños Cnerve no mielinizados que pueden identificarse en muestras histológicas de tanto las lesiones endometriales y del endometrio en sí a través detinción de histoquímica inmune. En contraste en mujeres no afectadas no hay fibras nerviosas dentro de las capas mucosas de endometrio. Si bien está clara distinción suena perfecta para una prueba de diagnóstico propuesta ha habido informes contradictorios donde algunos encuentran que la biopsia no es sensible o específica con un estudio retrospectivo que encontró el de 29 pacientes con adenomatosis sin endometriosis en la laparoscopia, el 69,2\% tenía tinción inmunohistoquímica neuronal positiva(Miller y Fraser 2015), sin embargo, no se encuentran la presencia de fibras neuronales de forma rutinaria enfermedad, inflamatoria pélvica(PID) o pólipos endometriales. Independientemente de esto, la presencia de estas nuevas fibras nerviosas aún se desconocen considerado clave en el dolor cíclico que las mujeres afectadas sienten como están sensibilizados a los estrógenos endógenos durante la menstruación donde a nivel tisular se produce inflamación. El tratamiento corriente de la endometriosis implica el uso de la combinación oral de anticonceptivos (COCP) y de hecho agonistas de GnRH que confirman que la endometriosis es una enfermedad sensible a las hormonas. Sin embargo, estos están plagados de efectos secundarios y no son ideales en esas mujeres quienes desean concebir (Hsu, y otros 2011). Es resultado de esto que los esfuerzos han sido hecho para detectar la actividad local de aromatasa, que puede reflejarse temprano en la enfermedad. Si bien esta sería una prueba muy útil, los primeros trabajos han 


\section{El papel de los biomarcadores en el diagnóstico precoz de la endometriosis}

Vol. 3, núm. 2., (2019)

Manuel Alejandro Cordones Sevillano; Kenya Andreina Recalde Dicado; Shirley Natali Lema Sarmiento; Alex Renato Quimis Moran

identificado que la expresión de aromatasa en mujeres infértiles no es diferente independientemente de si la causa es la endometriosis.

Sin embargo, como hay muchas proteínas reguladoras de esteroides que pueden estar implicado, puede ser que medir los niveles de enzimas localmente dentro del endometrio puede ser una herramienta de diagnóstico futura como lo será el mapeo expresión del receptor de estrógeno local en mujeres sintomáticas.

\section{Conclusión.}

En la actualidad, la endometriosis sigue siendo una condición altamente incapacitante, eso afecta a una gran cantidad de mujeres. Si bien se ha logrado mucho con respecto al tratamiento, el diagnóstico de esta afección permanece sin cambios desde décadas pasadas con muchas mujeres esperando años por un diagnóstico. Actualmente, varios métodos nuevos para identificar la etapa temprana de la enfermedad han sido identificada, aunque su significado sigue siendo desconocido. Además, si bien se ha realizado mucho trabajo en identificar biomarcadores en la endometriosis, uno de los principales problemas pocos han sido suficientemente estudiados para permitir el meta análisis y por lo tanto, cualquier conclusión que se pueda hacer. Para mejorar la utilidad de biomarcadores propuestos, se deben realizar estudios más grandes en pacientes según el subtipo de enfermedad y la presentación del paciente para permitir traducción significativa de datos experimentales a la clínica. 


\section{El papel de los biomarcadores en el diagnóstico precoz de la endometriosis}

Vol. 3, núm. 2., (2019)

Manuel Alejandro Cordones Sevillano; Kenya Andreina Recalde Dicado; Shirley Natali Lema

Sarmiento; Alex Renato Quimis Moran

\section{Bibliografía.}

A, Fassbender., RO Burney, T D’Hooghe, y L. Giudice. «Update on biomarkers for the detection of endometriosis. » Biomed Res Int, 2015.

Bolívar, J. Investigación Documental. México.Pax, 2015.

Borghese, B, KT Zondervan, MS Abrao, C Chapron, y D. Vaiman. «Recent insights on the genetics and epigenetics of endometriosis. » Clinical Genetics., 2016: 254-264.

Castro, J. Técnicas Documentales. México. Limusa, 2016.

Cosar, E, R Mamillapalli, GS Ersoy, S Cho, y B. Seife. « Serum microRNAs as diagnostic markers of endometriosis: a comprehensive array based analysis. » Fertil Steril, 2016: 402-409.

Das, PM, y JR. Bast. « Early detection of ovarian cancer. » Biomark Med, 2008: 291-303.

Davila, A. Concepto de terminos cientificos. Caracas: Oasis, 2015.

Grande, G, F Vincenzoni, D Milardi, G Pompa, y D. Ricciardi. «Cervical mucus proteome in endometriosis. 14: 7. » Clin Proteomics, 2017: 7-14.

Guerriero, S, S Ajossa, J Minguez, M Jurado, y V. Mais. «Accuracy of transvaginal ultrasound for diagnosis of deep endometriosis in uterosacral ligaments, rectovaginal septum, vagina and bladder. » Ultrasound Obstet Gynecol, 2015: 534-545.

Gupta, S, A Agarwal, L Sekhon, N Krajcir, Cocuzza, y M. «Serum and peritoneal abnormalities in endometriosis: potential use as diagnostic markers. » Minerva Ginecol, 2006: 527-551.

Hsu, AL, I Khachikyan, Stratton, y P. «Invasive and non-invasive methods for the diagnosis of endometriosis.» Clin Obstet Gynecol , 2011: 53.

Miller, EJ, y IS. Fraser. «The importance of pelvic nerve fibres in endometriosis.» Women's health , 2015: 611-618.

Mol, BW, N Bayram, JG Lijmer, MA Wiegerinck, y MY. Bongers. «The performance of CA-125 measurement in the detection of endometriosis: a meta-analysis.» Fertil Steril , 1998: 11011108.

Oliveria, M, T Raymundo, L Soares, T Pereira, y A. Demôro. «How to use CA-125 more effectively in the diagnosis of deep endometriosis. .» BioMed Research International, 2017: 6. 


\section{El papel de los biomarcadores en el diagnóstico precoz de la endometriosis}

Vol. 3, núm. 2., (2019)

Manuel Alejandro Cordones Sevillano; Kenya Andreina Recalde Dicado; Shirley Natali Lema Sarmiento; Alex Renato Quimis Moran

Randall, GW, PA Gantt, RL Poe Zeigler, CA Bergmann, y ME. Noel. «Serum antiendometrial antibodies and diagnosis of endometriosis.» Reprod Immunol, 2007: 374-382.

Riazi, H, N Tehranian, S Ziaei, E Mohammadi, y E. Hajizadeh. «Clinical diagnosis of pelvic endometriosis: a scoping review. .» BMC Womens Health, 2015: 15-39.

Tadros, M, y N. Keriakos. «Diffusion MRI versus ultrasound in How to cite this article: Dominic A W. The Role of Biomarkers in the Early Diagnosis of Endometriosis. .» Invest Gynecol Res Women's Health and Nuclear Medicine, 2016: 1765-1771.

$$
\text { @() }(\Theta()
$$

\section{RECONOCIMIENTO-NOCOMERCIAL-COMPARTIRIGUAL}

CC BY-NC-SA

ESTA LICENCIA PERMITE A OTROS ENTREMEZCLAR, AJUSTAR Y CONSTRUIR A PARTIR DE SU OBRA CON FINES NO

COMERCIALES, SIEMPRE Y CUANDO LE RECONOZCAN LA AUTORÍA Y SUS NUEVAS CREACIONES ESTÉN BAJO UNA LICENCIA CON LOS MISMOS TÉRMINOS. 